\title{
Tratamiento de contaminantes emergentes farmacologicamente activos por reacción Fenton y tratamiento biológico en reactor tipo Batch
}

\author{
Palomares-Reyna Daniela ${ }^{a}$, Flores-Valle Sergio Odin ${ }^{a}$, Mendoza-Pérez Jorge Alberto ${ }^{b *}$. \\ ${ }^{a}$ Escuela Superior de Ingeniería Química e Industrias Extractivas. Instituto Politécnico Nacional. Edificio No 7, Unidad Profesional \\ Adolfo López Mateos, Gustavo A. Madero, Lindavista, cp. 07738 Ciudad de México, México. \\ ${ }^{b}$ Escuela Nacional de Ciencias Biológicas. Instituto Politécnico Nacional. Av. Wilfrido M. Gustavo A Madero. Adolfo López Mateos CP. \\ 07738 Ciudad de México, México. \\ *E-mail: jorgemendozaperez@yahoo.com
}

Recibido 15 de octubre de 2016, Aceptado 15 de diciembre de 2016

\section{Resumen}

Está demostrada la presenci a de restos de productos farmacológi camente acti vos en las aguas resi duales, así como su baja eli mi nación durante los procesos de depuración convenci onales, exi sti endo estudios sobre los efectos acumulati vos y tóxi cos en los peces y otros ani males. Se ha estudiado el grado de eli mi nación de di sti ntos fármacos comunes como contami nantes emergentes medi ante tratami ento Fenton y tratami ento bi ológi co en un reactor semi conti nuo, debi do a su potencial tóxi co como di struptæes endócri nos. Los resultados globales fueron una degradaci ón superi or al 30\% en la mayoría de los fármacos, si endo los medicamentos en forma ai slada los mayormente eli mi nados. Para el caso del tratami ento bi ológi colos fármacos en mezcla interactúan entre ś́, pero la degradaci ón ocurre si n i mportar este hechø i ncluso con la mezcla farmacológi ca donde exi sti ó una degradaci óen la concentraci ón de la mi sma cercana al 77\%.Por otro lado, con el tratami ento Fenton los fármacos llegan a fragmentarse en un porcentaje mayor al $50 \%$ sin importar la ci néti ca de cada fármaco y en el caso de la mezcla de medi camentos, la eli mi nación en su concentración fue del $78.5 \%$ con el uso de alúmi na

Palabras clave: Tratamiento Fenton, Oxido-Reducción, Metabolismo, Fármaco, Aguas residuales.

\section{Introducción}

La preocupación por la exposición a disruptores endócrinos (EDC) y sus posibles impactos sobre la fauna y los seres humanos se ha vi sto incrementada durante la última década. Entre estas nuevas substanci as aparecen los PPCP's (fármacos y productos de hi gi ene personal), los cuales están di señados para ser muy acti vos e interaccionar con receptores específicos en el hombre y ani males. Después de su admi ni straci ón, los medicamentos después de ser absorbidos y metabolizados por el organismo, son excretados e incorporados a las aguas resi duales. Algunas de estas sustancias no son degradadas en las depuradoras y llegan a las aguas superficiales con los vertidos de los efluentes depurados [1].

Metabolismo de los fármacos: durante las reacci ones metabólicas existen dos fases: en la primera suelen existir oxidaciones, reducciones o hidrolisis que introducen en la estructura un grupo reactivo que lo convi erte en quími camente más activo, cambio de acti vi dad o inacti ka ón. En la segunda fase, ocurren conjugaci ones que por lo general i nacti van el fármaco [2].
En las aguas residuales se encuentran diferentes compuestos orgáni cos, entre ellos medi camentos que para su di sposi ción son tratados junto con los demás contami nantes. Algunas de las formas más usuales para la li mpi eza de las aguas residuales se llevan a cabo por medio de tecnologías biológicas (lodos acti vados), tecnologías avanzadas (uso de ozonoli si s, fotocatáli sis, bi orreactores de membranas, hi drólissi etc.), tecnologías combi nadas y finalmente, destacan los humedales arti fi ci ales y las plantas vgetales que son la base del proceso ya que degradan, absorben y asimilan en sus tejidos los contaminantes, pero tambi én proporci onan una extensa superfi ci e onde se favorece el creci mi ento bacteri ano y se reti enen los elementos sóli dos en suspensi ón $[3,4]$.

\section{Tratamientos biológicos}

Constituyen una serie de i mportantes procesos que ti enen en común la uti li zación de mi croorgani smos. Uno de estos procesos es el tratami ento de aguas resi duales En el metaboli smo bacteri ano, el elemento receptor de electrones juega un papel fundamental en los procesos de oxi daci ón de la materi a orgáni ca [4].

Las macromoléculas de los fármacos pueden inhibir el crecimi ento bacterial[5], sin embargo es posible 
que exista una degradación de los compuestos farmacológicamente activos por medio de tratami entos biológicos ya sea que se presenten en forma ai slada o en mezcla, para lo cual es necesario emplear pruebas de degradaci ón con lodos acti vados y de esta manera averi guar si por medi o ellos exi ste un comportami ento que impida o potencie la eli mi nación de los fármacos, especialmente si se encuentran en mezcla, debi do a que es de esta manera como usualmente se presentan en las aguas resi duales [6].

\section{Reacción Fenton}

El método se basa en la generación de radicales hi droxi lo por la adi ción de peróxi do de hidrogeno a sales metáli cas de hi erro en di soluci ónçon mejores resultados a un $\mathrm{pH}$ menor a 3 .

A la combinación de $\mathrm{H}_{2} \mathrm{O}_{2}$ y sales de hi erro se denomi na reacti vo Fenton[7].

Estos radicales $\mathrm{OH}$ son capaces de oxi dar muchos compuestos orgáni cos de forma no selectiva y con altas velocidades de reacción. Esta propiedad es aprovechada para conseguir la completa mineralización de ds contaminantes o su degradación en sustancias más fácilmente bi odegradables [8].

Se ha uti li zado el proceso de Fenton para reduci r la concentraci ón de materi a orgáni ca del efluente texti 1 en aguas residuales gracias a que por medio de la oxi daci óndestruye los enlaces pi en las moléculas conjugadas de los tintes, las cuales le confi eren la coloraci ón característi ca a cada uno[9,10].

Existiendo evidencia de que el tratami Ła Fenton puede romper enlaces pi en las moléculas conjugadas, el objeti vo de este trabajo es comprobar la efi cacia de la degradación de fármacos medante reacción Fenton y mediante tratami ento biológico en un reactor aerobi o ti po Batch, de esta manera se contemplaría el acoplami ento de los dos métodos para que la eli mi naci ó de los fármacos sea mayor.

Teni endo en cuenta las anteri ores ci rcunstanci as, se ha estudiado el grado de eliminación de distintos fármacos mediante tratami ento Fenton $\mathrm{y}$ tratami ento biológico en un reactor ti po Batch Estos fármacos fueron Tribedoce, Ibuprofeno, Paracetamol, Dropropi zi na,

Valproato, Ciprolisina, Dexne y Farmalor para el tratami ento biológico así como el uso de los primeros 6 medicamentos antes menci onados en conjunto con Hi drocorti sona para llevar a cabo el tratami ento con reacci ón Fenton. Los medi camentos
Hi drocorti sona, Dropropi zi na, Dexne y Farmalor se encontraban a una concentración del $10 \%$ en volumen, mi entras que el resto de fármacos se encontraban a $1 \%$. La elección de estos compuestos farmacológi camente acti vados se hi zo en bse a que son parte de los medicamentos de uso común mayormente encontrados en las aguas residuales [11-13].

\section{Parte experimental}

\subsection{Tratamiento de los fármacos en reactor biológico tipo Batch}

Para efectuar el estudio de la degradación de los fármacos se realizaron dos experimentos: en el primero se aislaron $30 \mathrm{ml}$ de cada uno de los medi camentos y en el segundo se tomaron $355 \mathrm{ml}$ de una mezcla de fármacos consti tui da porsoluci ones de Farmalor, Ci proli sina, Valproato, Paracetamol y Tribedoce a las concentraciones ya menci onadas; posteri ormente se colocaron en reactores ti po Batch junto con $5 \mathrm{ml}$ de lodos activados tomándose alícuotas de $8 \mathrm{ml}$ cada 30 minutos para su centri fugado a $3100 \mathrm{rpm}$ durante 10 mi nutos.

Para este tratami ento bi ológico se eligi eron 8 diferentes fármacos por ser algunos de los más comunes encontrados en las aguas residuales, los cuales fueron Tribedoce, Ibuprofeno, Paracetamol, Ciproli sina, Dexne, Troferit, Valproato, Farmalor y Ci prolisina.

\subsection{Tratamiento Fenton ácido}

El método de referenci a apli cado es el descri toen el artículo Treatment of Textile Dyeing Wastewater by Hydrogen Peroxi de and Ferrous Ions [14], método uti li zado para el tratami ento de colorantes produci dos por textiles dentro de las aguas residuales, es apropi ado tanto en condi ci ones aci das como bási cas, si n embargo se eli gi ó el medi o áci do para tratami ento Fenton en fármacos debi do a que en medi o bási co la coloraci ón produci da por los colorantes que conti enen algunos fármacos reaparece.

A conti nuaci ónse muestra la ruta desarrollada:

Se realizaron dos experimentos en donde en el pri mero, los 7 fármacos anteri ormente menci onados, se uti lizaron para completar un volumen de mezcla aproxi madamente de $400 \mathrm{ml}$.

El segundo experi mento se le reali zo a losfármacos Dropropizina e Ibuprofeno, elegidos de manera aleatoria con el fin de comparar la degradación en 
mezcla y de manera ai slada, en volúmenes de $15 \mathrm{ml}$ de soluci ón cada uno.

Posteri ormente a cada una de las soluciones se les apli có la reacci ón Fenton aci da, para lo cual se agregó $\mathrm{H}_{2} \mathrm{O}_{2}$ al $10 \%$ en volumen de soluci ón de cada fármaco y mezcla farmacológica, 10\% volumen de $\mathrm{FeSO}_{4}$ $0.008 \mathrm{M}$ y fi nalmente $0.3 \mathrm{ml} \mathrm{HCl}$ para acondi ci onar a un $\mathrm{pH}$ menor a 3 , manteni endo en reacción por 5 minutos con agitación constante. $\mathrm{La}$ reacción se di vi di ó en dos porci ones i guales: la pri mera se llevó a floculación agregando $1 \mathrm{~g}$ de alúmi na en agitación constante y se llevó a filtración mediante papel poroso. La segunda se fi ltró di rectamente.

Al fí nali zar la reacci ón se neutrali zæada soluci ón con $\mathrm{NaOH}$ concentrado hasta llegar a un $\mathrm{pH}=7$.

Finalmente tanto a las muestras tratadas con lodos activados y con reacción Fenton acida se les practicaron lecturas de absorbancia en un espectrofotómetro de luz UV-vi s compacto de 190 $1100 \mathrm{~nm}$.

\subsection{Parámetros de la Actividad Farmacológica (DQO)}

Para comprobar si los lodos acti vados y el tratami ento Fenton aumentaban la veloci dad de degradación de la materi a orgáni ca, se cuanti fi có la demanda quími ca de oxígeno. La demanda química de oxígeno (DQO), expresada en oxígeno, mide la porción de materia orgáni ca (M.O), bi odegradable o no, de una muestra que es susceptible de oxi daci ón por un fuerte oxi dante quími co (di cromato potási ce Cr2O7K2) [12].

\section{Resultados y discusión}

\subsection{Eliminación con el tratamiento biológico}

Para la prueba de degradación de los diferentes fármacos así como de la mezcla farmacológi ca después del tratamiento biológico, se realizó un análisis de absorbanci as medi ante espectro de luz UVvi s, en donde se presentan porcentajes de eliminación de la absorbanci a (decreci mi ento en la absorbanci a y aumento en su eli mi nación), obteni éndose las fi gura\$,2 y 3. Se puede apreciar que en la mayoría de los casos el porcentaje en la eliminación de las absorbancias aumentan con el transcurso del ti empo, a excepci ón de la mezcla farmacológica donde en el minuto 90 la eliminación disminuyepara posteriormente volver a aumentar, ya que la absorbancia se incrementa por un momento; lo mi smo sucede con el medicamento Farmalor donde el porcentaje de eliminación ti ende a disminuir. Este comportami ento se puede presentar probablemente debi do a la ci neti ca de cada fármaco.

Los resultados generales en el tratami ento bi ológico fueron una degradación máxi ma del Ibuprofeno con un porcentaje de eliminación del $37.24 \%$ al transcurrir 120min.; y una eliminación míni maen el fármaco Farmalor.

Algunos de los medicamentos presentan una cinética diferente y su coloración varía dependi endo el ti empo que tengan con los lodos.

Para la mezcla farmacológi ca el comportami ento en la eli mi nación es cambi ante, debi do a que esta mezcla se encuentra consti tui da por di ferentes grupos funci onales que al interactuar entre sí podrían generar nuevos productos, sin embargo de manera general si existe una eli mi naci ón o reducción en la absorbancia.

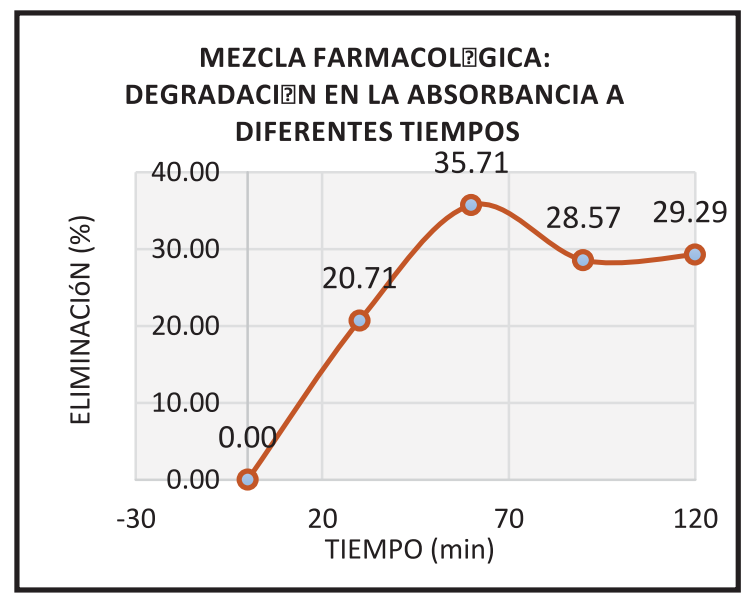

Figura 1. La tendencia de la mezcla se dirige al aumento en la eliminación de la absorbancia.

\section{DEGRADACI?N EN LA ABSORBANCIA A DIFERENTES TIEMPOS}

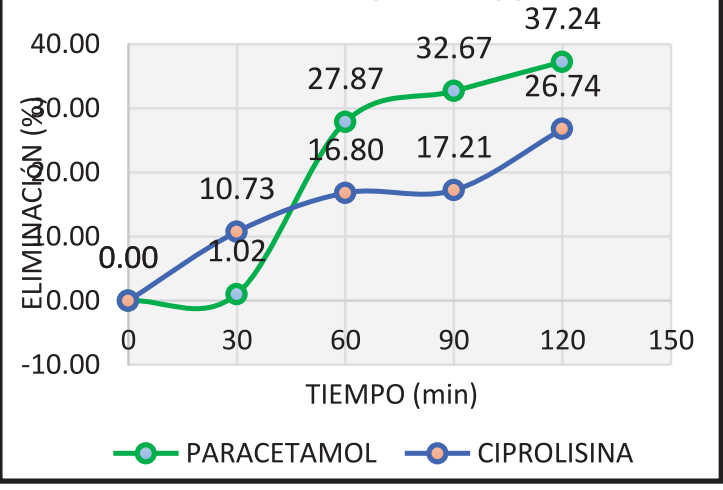

Figura 2. Eliminación vs tiempo en los fármacos Paracetamol (verde) y Ciprolisina (azul). 
DEGRADACIÓN EN LA ABSORBANCIA A DIFERENTES TIEMPOS

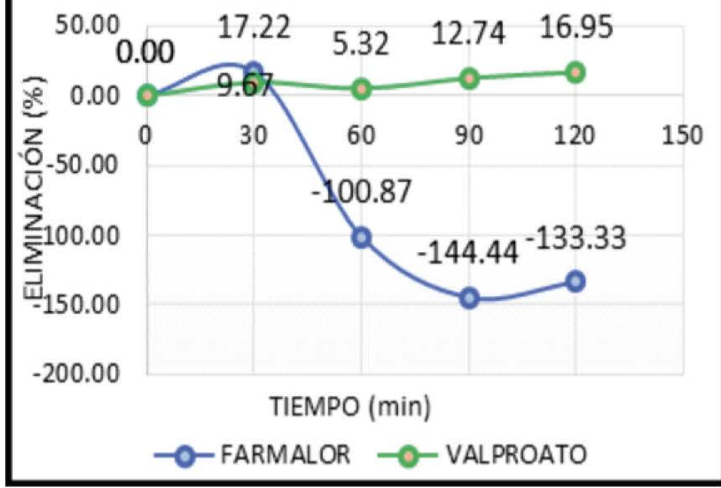

Figura 3. Eliminación vs tiempo en los fármacos Valproato (verde) y Farmalor (azul).

Para sustentar la información arrojada por la espectroscopia de luz UV-viş se realizaron las cuanti fi caci ones de la demanda quími ca de oxígende los di ferentes fármacos obteni éndose las fi guras $4,5 \mathrm{y}$ 6 donde se puede apreci ar como la concentraci ónde la materi a suscepti ble a ser oxi dada va decreci endo con el paso del ti empoen todos los medi camentos incluyendo al Farmalor y a la mezcla, a pesar de haber presentado vari aci ones en suabsorbancia comprobándose de esta manera que las bacteri as pueden metaboli zar productos farmacológi camente acti vos, muy a pesar de que estos estén di señados para i nhi bi r la acti vi dad bacteri ana

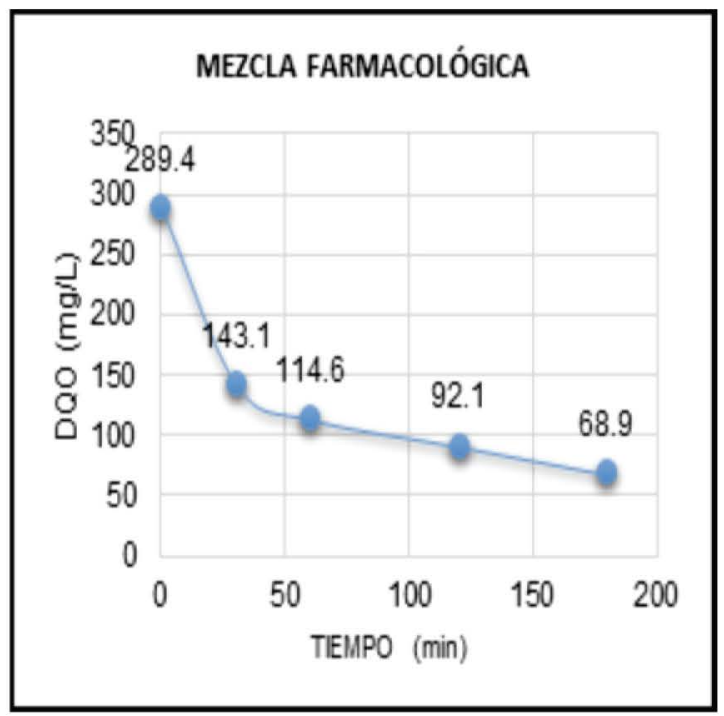

Figura 4. Degradación en la DQO de la mezcla farmacológica a diferentes tiempos.

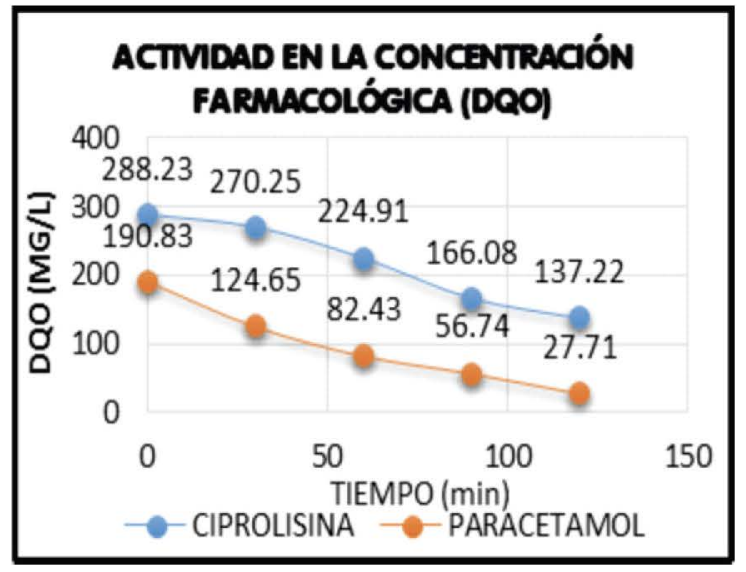

Figura 5. Degradación en la DQO en los fármacos Ciprolisina (azul) y Paracetamol (naranja) a diferentes tiempos.

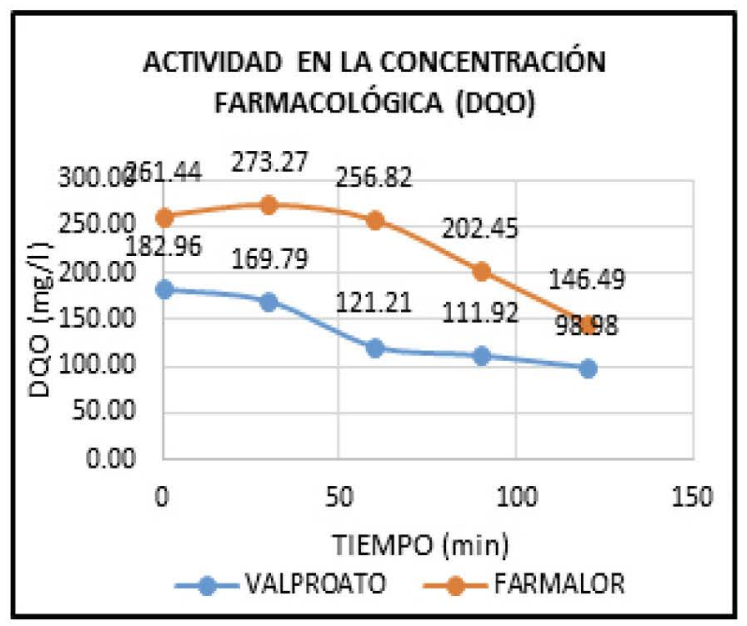

Figura 6. Degradación en la DQO en los fármacos Valproato (azul) y Farmalor (naranja) a diferentes tiempos.

La estructura molecular de cada uno de los fármacos es completamente diferente, por lo que sus grupos funci onales interactúan o responden de manera di sti nta al tratami ento en el reactor bi ológi co.

Por separado los compuestos activos de las muestras generan eliminación en lasabsorbancias que en la mayoría de los casos van aumentando paulati namente y no de manera súbi ta. Sin embargo en algunos fármacos esta absorbanci aes vari able

Durante el tratami ento bi ológi co de los fármacos las bacterias metabolizan fragmentos de los medi camentos generando $\mathrm{CO}_{2}, \mathrm{NH}_{3}$ y agua a partir de aquellas partes de la molécula en donde el carbón sea sencillo de separar [15]. Sin embargo exi sten ocasiones en donde la forma estructural de la molécula es tan compleja que puede interaccionar 
con otros compuestos generando un ti po de molécula di ferente.

Por ejemplo el fármaco Farmalor cuyo ingredi ente activo es la loratadina(fi g. 7), que es uno de los que menor grado de degradación tiene, en la cual se puede producir una hidroxilación ali fática $\mathrm{o}$ aromática, generando un alcohol que luego puede pasar a aldehído, todo esto graci as al interactuar con el oxígeno del medi o en el que se encuentra[16].

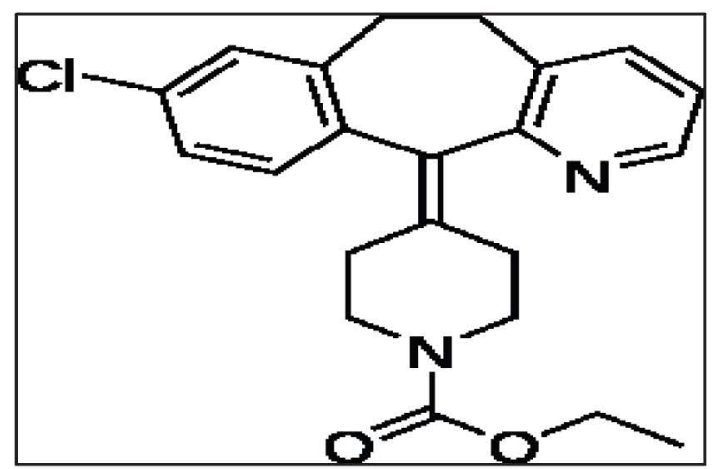

Figura 7. Molécula de la loratadina.

Las cargas más electronegati vas reti enen en mayor canti dad el haz de luz del espectro de luz vi sible, pero al encontrarse en conti nua resonanci a reestructuran nuevamente la molécula ini cial, logrando así que las absorbanci as en los ti empos i nci ales varíen.

Por otro lado, aquellos fármacos menos complejos y más hi drosolubles, como la molécula del Ibuprofeno (fig. 6), ti enden a ser metaboli zados más fáci lmente por las bacteri as [13], generando mayor cantidad de $\mathrm{CO}_{2}$, ya que es más factible que ocurra una desalqui laci ón, rompi endo la mlécula en partes más pequeñas.

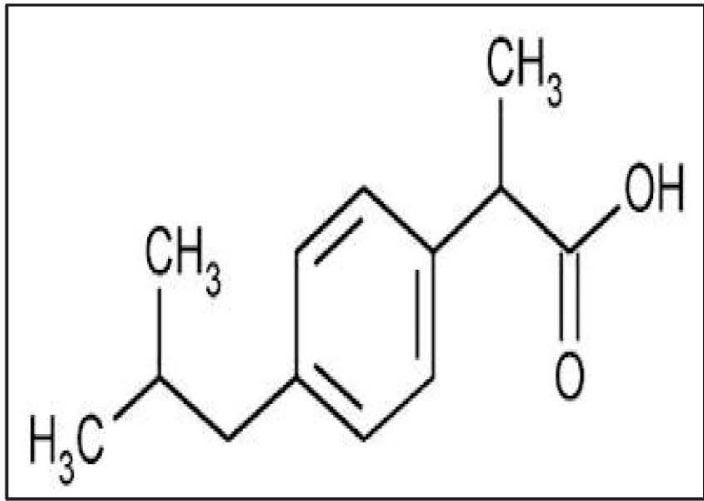

Figura 8. Molécula del Ibuprofeno.
Estas reestructuraci ones moleculares, el aumento en las cargas negati vas producto de la adi ci ón de oxígeno, el rompi mi ento de enlaces dobles, la desalquilación, así como la división de una molécula compleja en estructuras más pequeñas, provocan los cambi os en la absorbancia a distintas longitudes de onda, o que incluso las crestas que mostraba cada compuesto se vayan degenerando durante la lectura frente al espectroscopi o de luz vi si ble.

Cuando se presentan los fármacos en mezcla que es la forma más habitual en la que se encuentran en las aguas residuales, el comportami ento de la mezcla se encuentra li geramente cambi ante.

Estas variaciones pueden ser producto de la deslocalización de electrones en los enlaces "pi" de los compuestos aromáticos que puedan presentar las moléculas.

Se podría esperar que al encontrarse los fármacos en mezcla estos comi encen a reacci onar entre ellos, así como con las bacterias, generando diferentes metaboli tosy di ferentes gases.

Aun así parte de algunos componentes de cada fármaco no podrán ser degradados en su totali dad y se habla de que estas sean las trazas de contaminantes que posi blemente pudi eran quedar en las aguas subterráneas y/o superficiales, ya que con el paso del ti empo la eliminación se va haci endo cada vez míni mhasta llegar a un punto donde se vuelva casi constante, por lo que una recomendación seria complementar el tratami ento biológico con mi cro o nano membranas para la total retenci ón de estos contami nantes.

\subsection{Eliminación con el tratamiento Fenton:}

Esta reacción oxidereducción se le realizó a los fármacos Paracetamol, Dropropi zi nay a una mezcla farmacológi ca de medi camentos a concentraci ones ya menci onadas, en volúmenes de muestra de $30 \mathrm{ml}$, di vi di éndolos en dos partes iguales con la finalidad de aplicarles el tratamiento con y sin ayuda de coagulaci ón por medi o de alúmi na.

A estos fármacos, incluida la mezcla, s les practi caron di luci oneshasta completar 4 volúmenes, cada uno si endo el 50\% del anteri or, desde un $100 \%$ de concentración hasta un $6.25 \%$, con el fin de crear una curva de cali braciónen un espectro de luz UVvi s a sus respecti vas longi tudes de ondadentro de la cual se pudiera interpolar el valor de absorbancá arrojado en el tratamiento de cada fármaco y por medio de regresión lineal y con ayuda de la absorbancia se encontrara el porcentaje de concentración del fármaco posteri or al tratami ento 
Fenton, o bi en se compara como fue que la mi sma cantidad de volumen ahora mostrara una reducci ón considerable en la absorbancia Los resultados se presentan en las fi guras 9,10 y 11 , donde se observa como a partir de una curva de calibración con porciento en concentración inicial (punto de absorbancia más alto en la gráfica) posterior al tratami ento de oxidación Fenton, la absorlancia decrece considerablemente en cada fármaco, i ncluyendo la mezcla

Los resultados del tratami ento Fenton demuestran cómo esta reacci ón logra fragmentar las moléculas de cada uno de los fármacos, sin embargo el uso de alúmi na no si empre benefi ci a este fenómeno, ya que si el fármaco se encuentra solo, como es el caso del Ibuprofeno o la Dropropi zi na, la absorbanci a ti ende a aumentar, debido a que las concentraciones en la soluci ón son tan pequeñas que las partículas no son sufi ci entes para lograr una coagulaci ón completa. En cambio, en el caso de la mezcla de medi camentos la concentración es mayor por lo tanto y por di ferencia de cargas, el alumi ni o puedecaptar mayor canti dad de partículas y por lo tanto coagular y sedi mentar por gravedad la mayor cantidad del complejo, logrando que de esta forma el fi ltrado sea más exi toso.

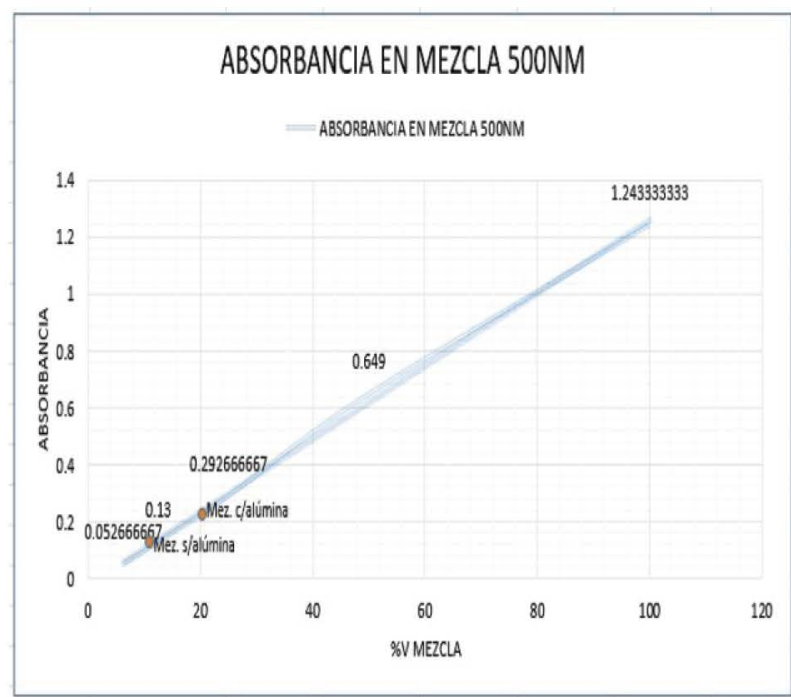

Figura 9. Curva de calibración y resultado del tratamiento Fenton en mezcla: en puntos naranjas se puede observar las coordenadas del resultado post oxidación con y sin alúmina, siendo resultados menores a la dilución 3 y 4 de la curva.

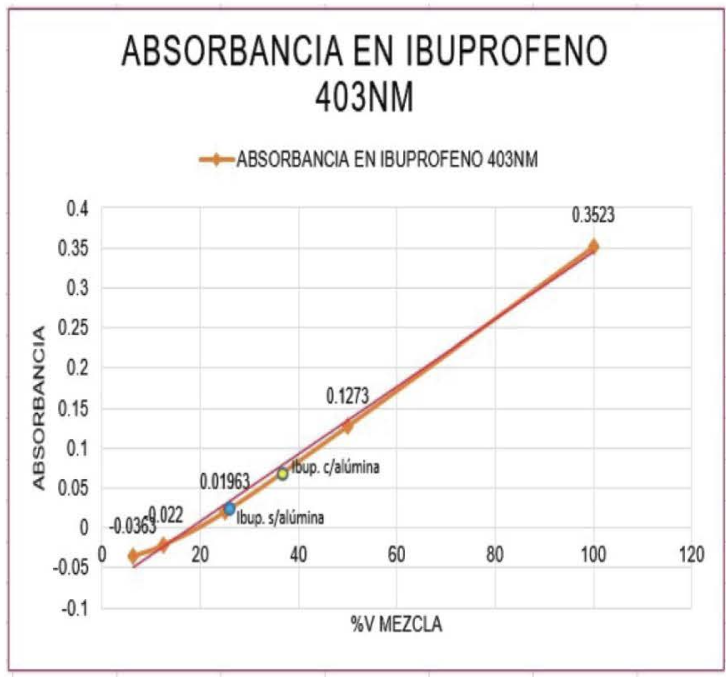

Figura 10. Curva de calibración y resultado del tratamiento Fenton en Ibuprofeno a $403 \mathrm{~nm}$ : en el punto azul se puede observar la coordenada del resultado post oxidación sin uso de alúmina y en amarillo la coordenada con alúmina.

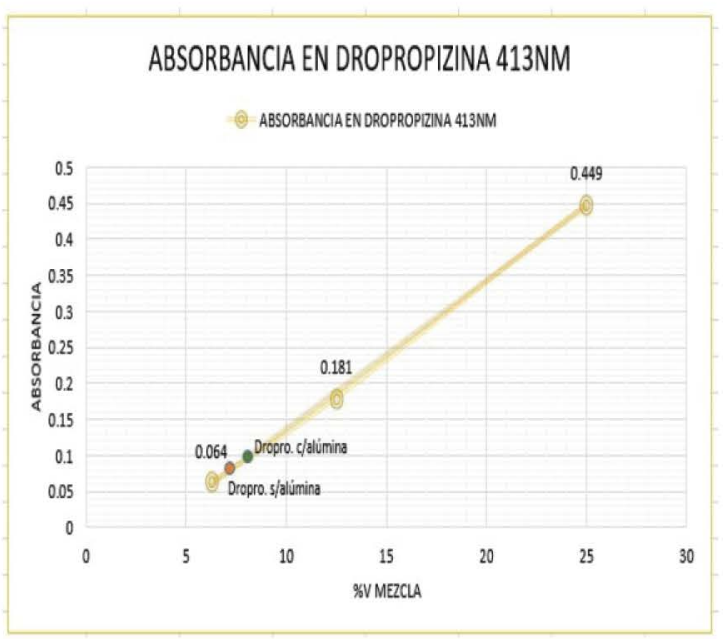

Figura 11. Curva de calibración y resultado del tratamiento Fentón en Dropropizina a $413 \mathrm{~nm}$ : en el punto naranja se puede observar la coordenada del resultado post oxidación sin uso de alúmina y en verde la coordenada con alúmina, siendo resultados menores a la dilusión 3 de la curva.

Es necesari o hacer una comparaci ón de los resultados anteri ores con los datos de la demanda quími ca de oxígeno (DQO) que se presentan en las fi guras 12, 13 y 14 las cuales demuestran cómo cada fármaco ai slado así como la mezcla, van di smi nuyendo su concentración al aplicarles la reacción de óxido reducci ón Fenton, habi endo uti li zado o no la alúmi na. Sin embargo es notori o que el uso de este coagulante ayuda a aumentar la eli mi nación de los fármacos en las di luci ones, si endo el Ibuprofao el que presenta 
un mayor grado de degradación, presentando una concentraci ón de 26.9 ppm.

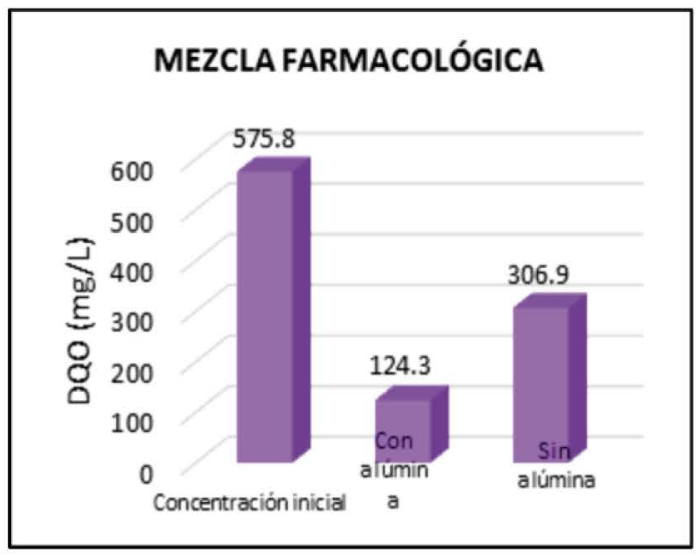

Figura 12. Degradación en la concentración de la mezcla farmacológica posterior al tratamiento Fenton con y sin alúmina.

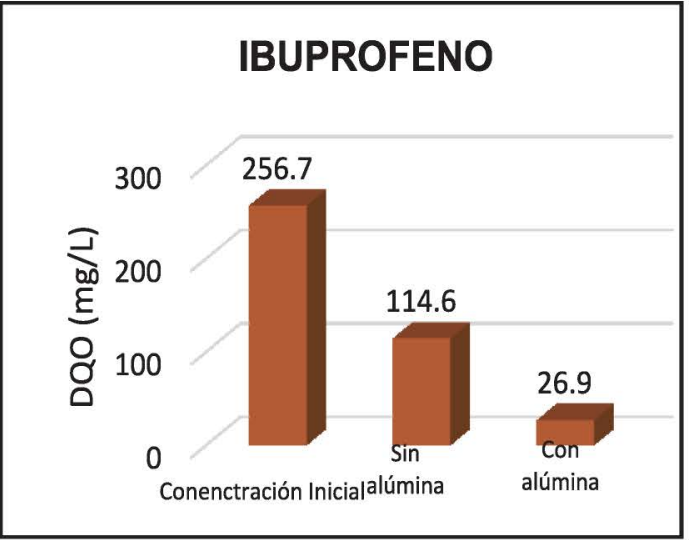

Figura 13. Degradación en la concentración del fármaco Ibuprofeno posterior al tratamiento Fenton con y sin alúmina.

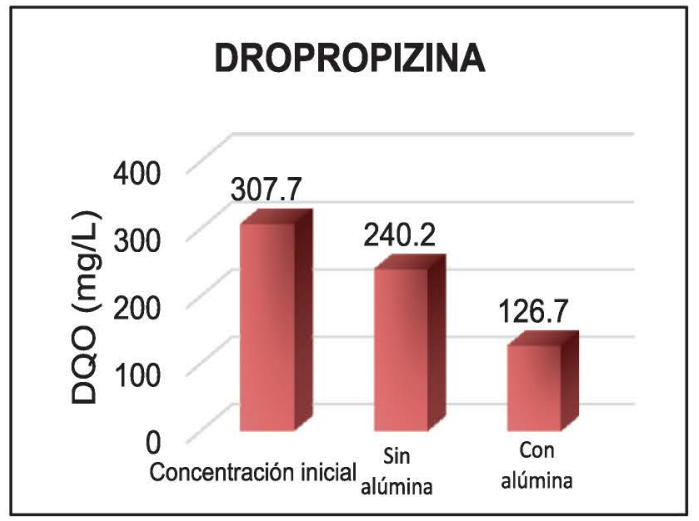

Figura 14. Degradación en la concentración del fármaco Dropropizina posterior al tratamiento Fenton con y sin alúmina.
La coloración y los agentes perfumantes son componentes que en los fármacos son algunos de los primeros en desaparecer, e incluso cuando se encuentran en mezcla se puede observar una decoloraci ón gradual de las soluci ones ini ciales fig. 15).

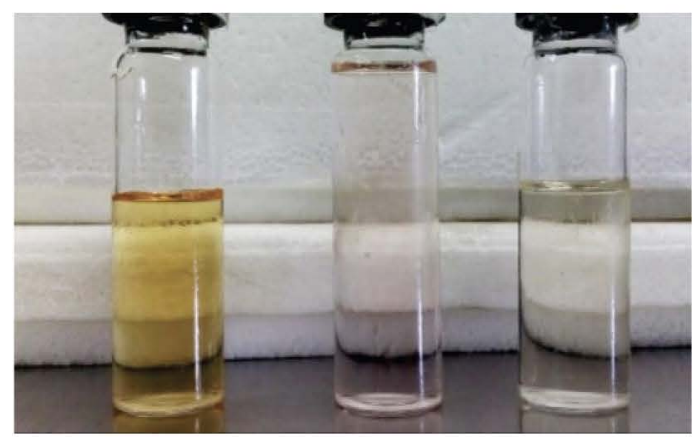

Figura 15. Decoloración de la Dropropizina antes del tratamiento Fenton (Izquierda) y después del tratamiento sin (al centro) y con Alúmina (derecha).

Finalmente, si bien es cierto queen el tratami ento bi ológico algunos de los fármacos presentan comportami entos variados, reestructurando sus moléculas y generando reacciones secundarias aun a pesar de que los medicamentos estén di señados para inhibir el proceso metabólico de las bacterias, el tratami ento bi ológi co logra di smi nuir la comtraci ón de los fármacos. Sin embargo, el tratami ento Fenton logra ser más exi toso ya que la ci néti ca vari able de cada fármaco no es problema para que exi sta una eli mi naci ón en la concentración de cada medi camento, además de que es posible que se conti núecon la degradación del mismo si se le aplica nuevamente la reacción de oxi daci ón.

\section{Conclusiones}

Las sustancias farmacológicamente acti vas incluyen compuestos formados por moléculas complejas, que están di señadas para i nhi bir la acti vi dad bacteri ana, por lo que el tratami ento biológico a pesar de ser una alternativa viable para la eli minación de fármacos y contaminantes en general, puede no ser demasiado efici ente, ya que con el ti empo la degradación de los medi camentos se vuelve casi constante y pueden quedar trazas del fármaco.

La degradaci ón de contami nantes emergentes en reactor bi ológico se observó que tiende a ser selectivay que muchas veces se encuentra en constante variación, especialmente durante los primeros minutos de ser incorporada al reactor 
El tratami ento de óxi do-reducción Fenton tiene la capaci dad de eliminar los fármacos no i mportando su acti vi dad ci néti ca o estructura molecularpor lo que se presenta como una alternati va vi able para la depuración de agua.

El uso de coagulantes como la alúmi na en el tratami ento Fenton ayuda a disminuir la concentración de los contaminantes en un porcentaje mayor al $50 \%$. Al no usarse este aditivo también exi ste una eli mi nación de fármacos por medi o de fi ltrado, si n embargo para que se eli mi naran por competo se necesi taría repeti $r$ el proceso en mayor número de veces que uti li zando alúmi na.

El tratami ento Fenton resulta ser más efi ci ente y rápi do para la eli mi naci ón de los fármacos en comparci ón del tratami ento bi ológi co cuya eli mi nación ti ende a ser más selectiva.

\section{Referencias}

1. Falero, M. A.; Celso, P. B.; Fonseca, M. CENIC Cien. Biol. 2005, 26, 72-77.

2. Cortacans, T. J.; Hernández, L. A.; Del Castillo, G. I.; Carmona, M. E.; Hernández, M. A. Presencia de fármacos en aguas residuales y eficacia de los procesos convencionales en su eliminación. Congreso de ingeniería Civil y medio ambiente, Universidad Politécnica de Madrid, Dpto. Ordenación del Territorio, Urbanismo y Medio Ambiente, 24 de Octubre de 2006.

3. Miceli-Montesinos, A. S.; Nájera, A. H.; Rojas, V. M.; Quintero, L. L.; Orantes, G. C. Esp. I+D. 2014, 3(5), 73-95.

4. Rodríguez, F. A.; Letón, G. P.; Rosal, G. R.; Dorado, V. M.; Villar, F. S.; Sanz, G. J. Tecnologías convecionale. En tratamientos avanzados de aguas residuales industriales, 2da edición, Elecé Industria Gráfica.; Madrid, 2005; pp 18-43.

5. Farmacología II. Inhibidores de la bared bacteriana https://farmacomedia.files.wordpress.com/2010/05/inhibidoresde-la-pared-bacteriana.pdf (accesado el 24 de agosto de 2016). 6. Gil, M. J.; Soto, A. M.; Usma, J. I. Prod. + Limp. 2012, 7, 52-63.

7. Rubio, C. A.; Chica, E. L.; Pañuela, G. A. Ing. y Comp. 2012, 16(2), 211-223.

8. Blanco, J. J. Degradación de un efluente textil real mediante procesos Fenton y Foto-Fenton. Tesis de Maestría, Universidad Politécnica de Catalunya, Barcelona, PA, Julio de 2009.

9. Castro, P. L.; Durán, H. J.Tec. en Mar. 2013, 27(2), 40-50.

10. Definición y características más importantes de los colorantes. http://www.analizacalidad.com/docftp/fi192col.pdf (accesado el 17 de octubre de 2016).

11. Quesada, P. I. Traitements d'eau polluee par des produits pharmaceutiques. Tesis de Doctorado, Université de Toulouse, Toulouse, Francia, Marzo de 2009.

12. Hernández, I.; Alejo, K. M.; Méndez, L.; García, A.; Cordova, A.; García, A. Avan. En Cien. e Ing. 2015, 6(3), 1-8.

13. Gómez, P. C. Eliminación de Tetraciclinas de las aguas mediante procesos avanzados de oxidación. Tesis de Doctorado, Universidad de Granada, Granada, Mayo de 2011.
14. Perkowski, J.; Lech, K. Fib. \& Text. in East. Eu. 2002, 15, 78-81.

15. Fisiologia y metabolismo bacteriano. http://www.higiene.edu.uy/cefa/Libro2002/Cap\%2011.pdf (accesado el 5 de diciembre de 2016)

16. El metabolismo de fármacos, generación de metabolitos reactivos y su papel en el origen de las reacciones inmunológicas a fármacos. http://www.uv.es/jcastell/Metabolismo_de_farmacos.pdf (accesado el 5 de diciembre de 2016) 